\title{
Investigation of Liquid Diffusion into Contact Lenses Using an Electron Spin Resonance Technique
}

\author{
Evrim E. Özgür, ${ }^{1}$ Emine Aydin, ${ }^{2}$ Turan Özbey, $^{1}$ Murat İrkeç, $^{3}$ Banu Bozkurt, ${ }^{4}$ H. Yılmaz Kaptan ${ }^{1}$ \\ ${ }^{1}$ Hacettepe University, Department of Physics Engineering, Beytepe, Ankara, 06800, Turkey \\ ${ }^{2}$ Turkish Atomic Energy Authority, Ankara Nuclear Research and Training Center, Beşevler, Ankara, Turkey \\ ${ }^{3}$ Hacettepe University, Department of Ophthalmology, Sihhiye, Ankara, 06100, Turkey \\ ${ }^{4}$ Specialist in Ophthalmology, Private Practice, Turkey
}

Received 18 June 2004; accepted 28 March 2005

DOI 10.1002/app.22537

Published online in Wiley InterScience (www.interscience.wiley.com).

\begin{abstract}
In this study, electron spin resonance (ESR) spectroscopy was used for the first time to investigate liquid diffusion into contact lenses. As contact lenses are not paramagnetic substances, they were labeled with nitroxide spin probes to get an ESR spectrum. Thus, it gives a solid spinlabeled ESR spectrum. The shape and intensity of the ESR signals depend on the environment of these spin probes. The spin probe environment began to change from solid to liquid if liquid were dropped into the system. Consequently
\end{abstract}

the ESR spectra began to change with time, too. By following these changes, three distinct steps were found. Their diffusion coefficients were determined to be $6.38 \times 10^{-8} \mathrm{~cm}^{2} / \mathrm{s}$ for the first step (rapid decay region) and $0.37 \times 10^{-8} \mathrm{~cm}^{2} / \mathrm{s}$ for the second step (slow decay region), and $2.50 \times 10^{-8}$ $\mathrm{cm}^{2} / \mathrm{s}$ for the third and last step (desorption region). (C) 2006 Wiley Periodicals, Inc. J Appl Polym Sci 100: 2942-2946, 2006

Key words: ESR; hydrogels; diffusion

\section{INTRODUCTION}

The production of contact lenses is a modern technological breakthrough, and this branch of science is still undergoing tremendous growth. As all contact lenses consist of amorphous polymer chains, the type of monomer used can significantly change the property of the lenses. The primary qualifications of a good contact lens polymer are optical transparency; softness; high permeability to oxygen, nitrogen, carbon dioxide gases and water; wettability; and thermal and chemical stability.

The cornea is supplied with oxygen mainly from atmospheric oxygen because it does not have a vascular supply to take up $\mathrm{O}_{2}$ and $\mathrm{CO}_{2}$. Thus, the permeability of contact lenses to oxygen and liquid is an important parameter. If a lens does not provide adequate permeability, the eye suffers serious health risks. ${ }^{1}$

Electron spin resonance (ESR) spectroscopy is used in the investigation of paramagnetic materials; hence, there is a need for unpaired electrons. Contact lenses produced from special polymeric materials generally show a diamagnetic property. Therefore, polymeric environments can be made sensitive to the ESR technique by breaking bonds with radiation or inserting unpaired electron-carrying molecules.

Bonding of unpaired electron-carrying organic molecules to macromolecules is called labeling, and

Correspondence to: H. Y. Kaptan (kaptan@hacettepe.edu.tr).

Journal of Applied Polymer Science, Vol. 100, 2942-2946 (2006) (C) 2006 Wiley Periodicals, Inc. the bonding molecule is referred to as the spin label. Polymer studies generally have used nitroxide spin labels. The properties of nitroxide spin labels (such as being stable in liquid and solid environments, being soluble in polar and nonpolar liquids, and having simple fine-structure interaction) make them suitable for use in ESR techniques. ${ }^{2-6}$

The diffusion mechanisms and kinetics of polymers have been studied both theoretically and experimentally in our laboratories. In those studies, we examined the diffusion coefficients of some polymers by using ESR. ${ }^{7-10}$ In our previous works we used poly(methyl methacrylate), poly(vinyl acetate), poly(methacrylonitril), and polypropylene (PP). And we developed the theory of diffusion coefficients $(D)$ in spherical particles cylinders, and thin sheets, and we also determined $D$ values by using the relation between radical concentration $\left(R_{t}\right)$ and time $(t)$.

Because the samples we used in the present study were thin sheets, a brief theoretical outline relating $R_{t}$ to $D$ follows.

In this work diffusion is assumed to be Fickian, with a constant diffusion coefficient, $D$. The diffusion equation of Fick's second law ${ }^{11}$ is

$$
\frac{\delta C}{\delta t}=D \nabla^{2} C
$$

where $C$ is the concentration of the diffusing substance in the plane sheet. In this study it was assumed that the shape of the contact lens was a plane sheet. The 
result of Crank' ${ }^{11}$ solution for this equation in a plane sheet of the sample thickness, $\ell$, is given by eq. (2).

$$
\frac{M_{t}}{M_{\infty}}=1-\frac{8}{\pi^{2}} \exp \left(-\frac{D \pi^{2} t}{4 l^{2}}\right)
$$

where $M_{t}$ and $M_{\infty}$ are the amounts of diffusant entering the plane sheets at times $t$ and infinity, respectively. ${ }^{1,12}$

This equation forms the theoretical basis for determining $D$ in this study. Similar equations are also derivates and are used for other geometries, such as cylinders and spheres. ${ }^{10,13-15}$

It is known that ESR lines give the radical concentration, $R$, not the amount of diffusant, $M$. So the relation between the ratios $M_{t} / M_{\infty}$ and $R_{t} / R_{0}$ needs to be determined. $R_{0}$ and $R_{t}$ are the primary radical concentrations at $t=0$ and at time $t$, respectively. In our system there is a direct relation between the measured quantity $R$ (signal intensity) and $M$.

$$
\begin{gathered}
\frac{R_{t}}{R_{0}}=\frac{M_{t}}{M_{\infty}} \\
1-\frac{R_{t}}{R_{0}}=\frac{8}{\pi^{2}} \exp \left(-\frac{D \pi^{2} t}{4 l^{2}}\right)
\end{gathered}
$$

It is possible to follow and save the ESR signal intensities, $R$, with time by using an ESR spectrometer. Using this data, the diffusion coefficient, $D$, can be determined from the slope of the graph of $\ln \left(1-R_{t} /\right.$ $R_{0}$ ) versus time $t$ if the thickness of the sample, $\ell$, is known.

\section{EXPERIMENTAL}

All the hydrogel contact lenses used in this study were made of etafilcon-A [2-hydroxyethyl methacrylate (HEMA) polymer with sodium methacrylate and 2-ethyl-2-hydroxymethyl-1,3-propanediol trimethacrylate] with an average thickness of $0.07 \mathrm{~mm}$. We used

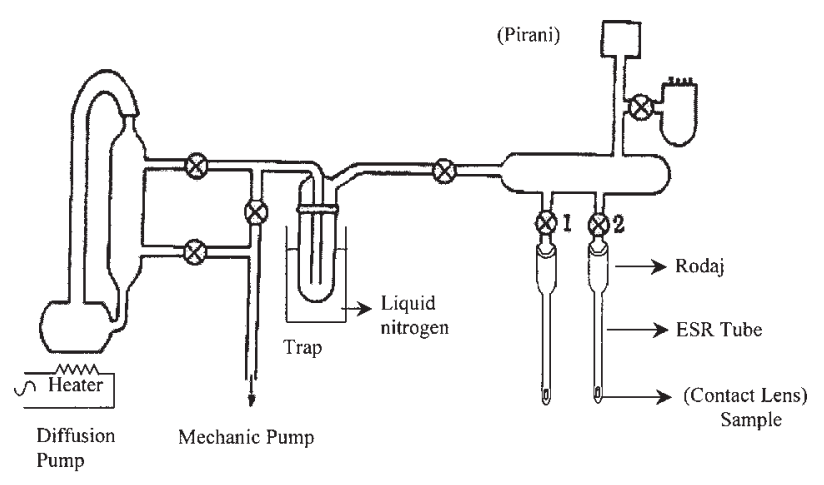

Figure 1 Vacuum system.

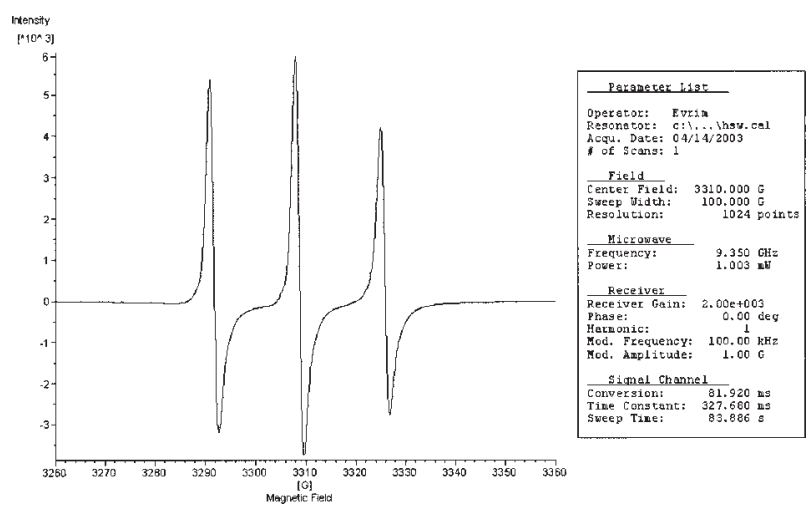

Figure 2 One of the original ESR spectra of a sample.

2.2,6.6-tetramethyl-1-piperidinyloxyl (TEMPO) nitroxide spin probes.

To prepare a $40-\mathrm{mM}$ stock solution, $0.156 \pm 0.015 \mathrm{~g}$ of TEMPO spin label was dissolved in $25 \mathrm{~mL}$ of distilled water. After a contact lens was put into a Pyrex ESR tube, stock solution was added to tube, and it was incubated in this solution for 3 days at room temperature. After this, the contact lens was cleaned with distilled water and was vacuumed under a $10^{-3}$ torr pressure for $30 \mathrm{~min}$ (Fig. 1). At the end of the whole process, the contact lens was a spin-labeled contact lens (SLCL).

The ESR spectrometer that we used was a Bruker EMX-131 $\times$ Band Spectrometer. The magnetic center field was $3310 \mathrm{G}$, the sweep width was $100 \mathrm{G}$, and the sweep time was $84 \mathrm{~s}$. The microwave frequency was approximately $9.3 \mathrm{GHz}$. The time constant was 327.68 ms, the modulation amplitude was $1 \mathrm{G}$, and the microwave power was kept at $1 \mathrm{~mW}$ throughout the study. These values are shown in Figure 2.

In this study a multipurpose contact lens solution was used as the liquid for diffusion into the contact lenses. First, an ESR spectrum of SLCL was obtained

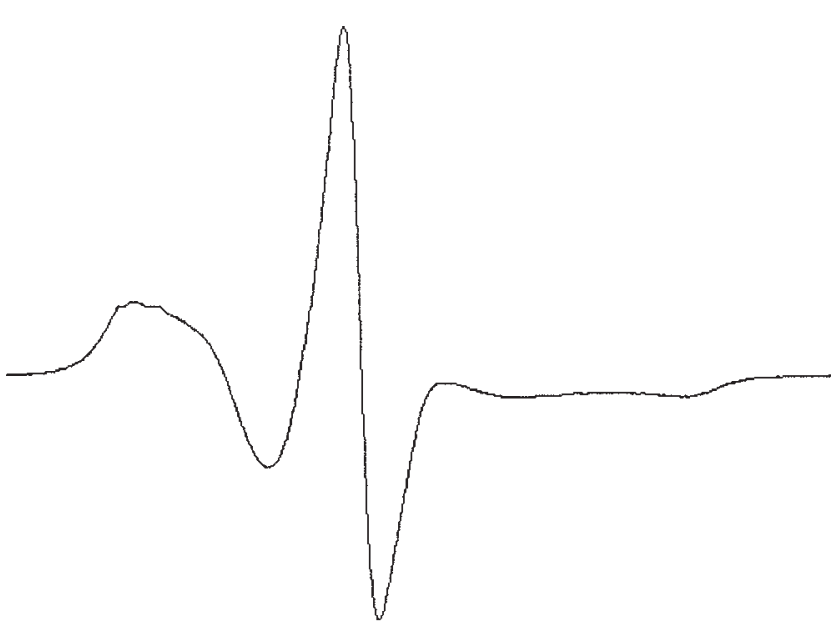

Figure 3 ESR spectrum of solid spin-labeled system. 


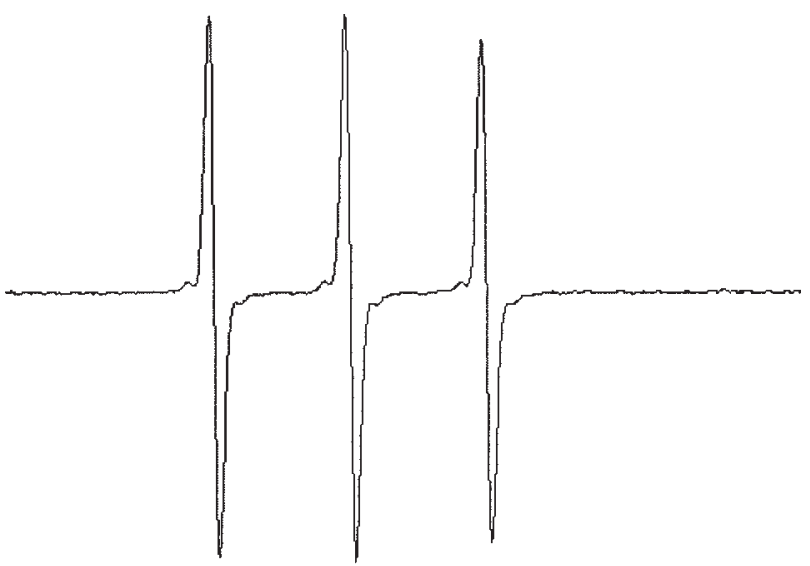

Figure 4 ESR spectrum of spin-labeled system in a liquid.

at time $t=0$. After obtaining this first spectrum, a multipurpose contact lens solution was added to the ESR tube that contained the SLCL. ESR spectra were recorded for $5 \mathrm{~h}$ at 2- to 5-min intervals.

\section{RESULTS AND DISCUSSION}

Nitroxide radicals labeled into the contact lenses were the source of the ESR signals of our sample. And the shape and intensity of the lines were strongly related to their environment. A typical ESR spectrum of the solid spin-labeled system is shown in Figure 3. And an ESR spectrum of the spin-labeled system in a liquid is given in Figure 4. From Figures 3 and 4 it can be clearly seen that the ESR spectra of solid and liquid systems are different in shape; therefore, it was possible to identify solid or liquid phases by their ESR signals. ${ }^{16,17}$

In Figure 5 only four spectra were chosen out of approximately 70 recorded during a 5 -h period. So it is fair to say that Figure 5 summarizes our experiments.

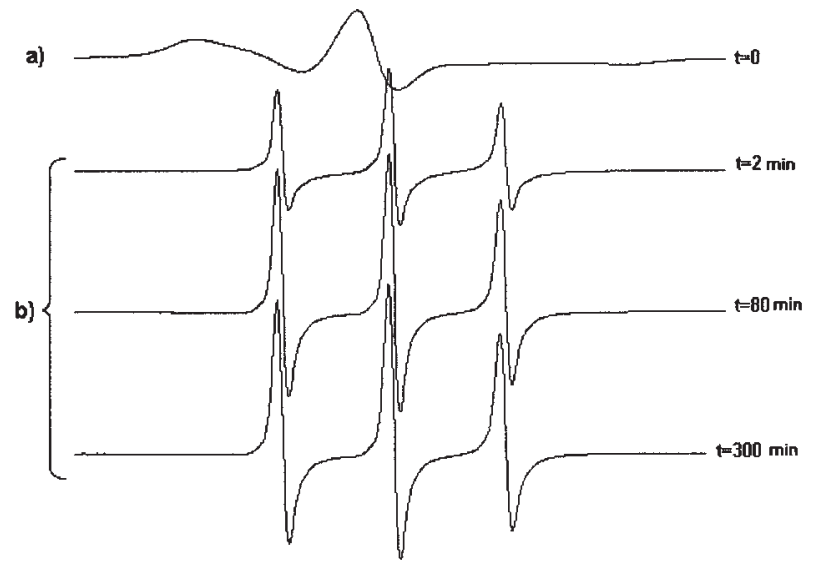

Figure 5 Change of the ESR spectrum of SLCL in liquid with time.

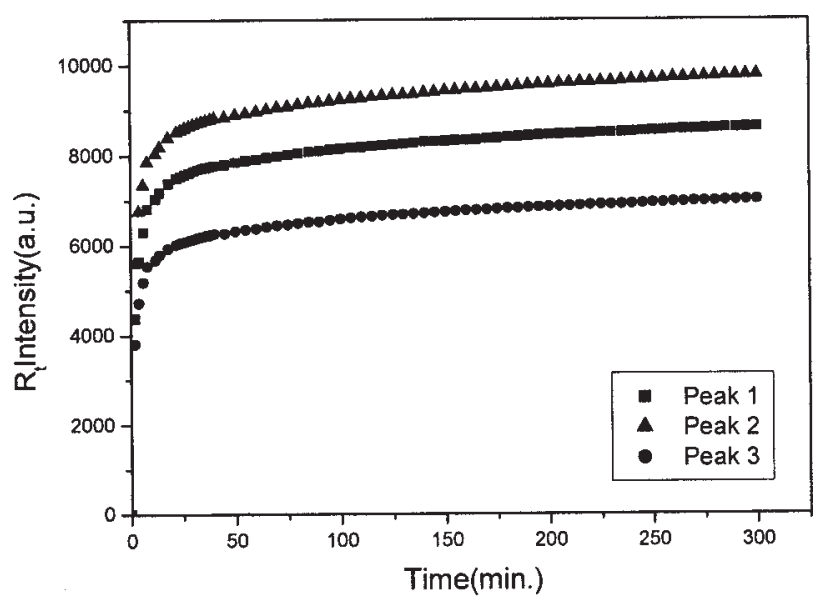

Figure 6 Signal intensity as a function of time for three peaks.

The ESR signal shown in Figure 5(a) is the first spectrum; it was taken at time $t=0$, when there was no solvent in the ESR tube. As only solid SLCL was present in the tube at this stage, the environment of the radicals was solid and their motion was restricted. Therefore the spectrum came from the labeled radicals in the lens.

When the liquid was dropped onto the lens, it diffused into SLCL via pores, so that liquid began to collect around radicals. In this case, the recorded ESR spectrum was the result of the overlapping of solid and liquid spectra. As long as liquid diffused into the lens, the number of radicals immersed in liquid increased. Consequently, the ESR lines representing intensity of the liquid increased, whereas the ESR lines representing intensity of the solid decreased. These changes can be seen in Figure 5(b).

We were able to draw a graph of $R_{t} / R_{0}$ versus time $(t)$ by using all the saved data of the three peaks in our

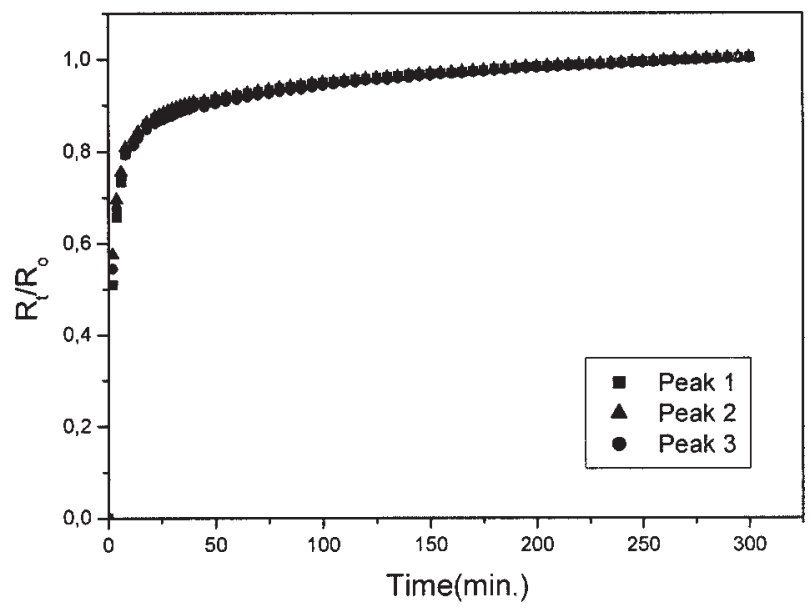

Figure $7 R_{t} / R_{0}$ values as a function of time for three peaks. 


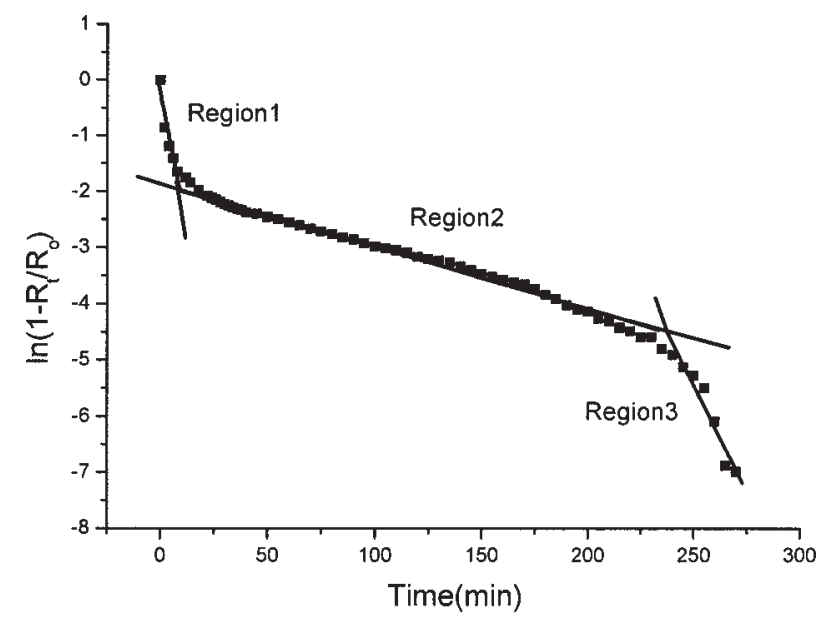

Figure 8 Graph of $\ln \left(R_{t} / R_{0}\right)$ versus time for second peak.

ESR spectra. We used intensity for $R_{t}$ because the line-width $\Delta H_{P P}$ remained constant during the experiment. The change in intensity with time for all three peaks can be seen in Figure 6 .

Changes in $R_{t}$ values normalized to $R_{0}$ with time are shown in Figure 7. In this graph, it can be seen that three peaks fit with each other. So the diffusion coefficients calculated from the lines should be equal. Therefore, we preferred to calculate the diffusion coefficient by using the second peak value only.

According to eq. (4), the changing of $\ln \left(1-R_{t} / R_{0}\right)$ with time $(t)$ should give a linear relation. This curve is shown in Figure 8, in which it can be seen that there are three linear regions with different slopes. The $D$ values, which are shown in Table I, were calculated by using these slopes and eq. (4).

As the $D$ values were not the same, it can be said that there are three different motions of liquid in the lens.

In the first region, that is, the first $10-15 \mathrm{~min}$, the liquid diffused into the solid system easily because there was no resistance to it. It can be clearly seen in Table I that the biggest $D$ value, $6.38 \times 10^{-8} \mathrm{~cm}^{2} / \mathrm{s}$, belonged to this region.

The second region was between 15 and $200 \mathrm{~min}$. And the $D$ value of this region, $0.37 \times 10^{-8} \mathrm{~cm}^{2} / \mathrm{s}$, was less than that of the first region, possibly because the liquid molecules that entered behaved as a barrier. This hindered the penetration of new sol-

TABLE I

Diffusion Coefficient Values of Liquid into Contact Lens

\begin{tabular}{lcc}
\hline & $D\left(\mathrm{~cm}^{2} / \mathrm{s}\right)$ & \\
\hline Region 1 & Region 2 & Region 3 \\
\hline $6.38 \times 10^{-8}$ & $0.37 \times 10^{-8}$ & $2.50 \times 10^{-8}$ \\
\hline
\end{tabular}

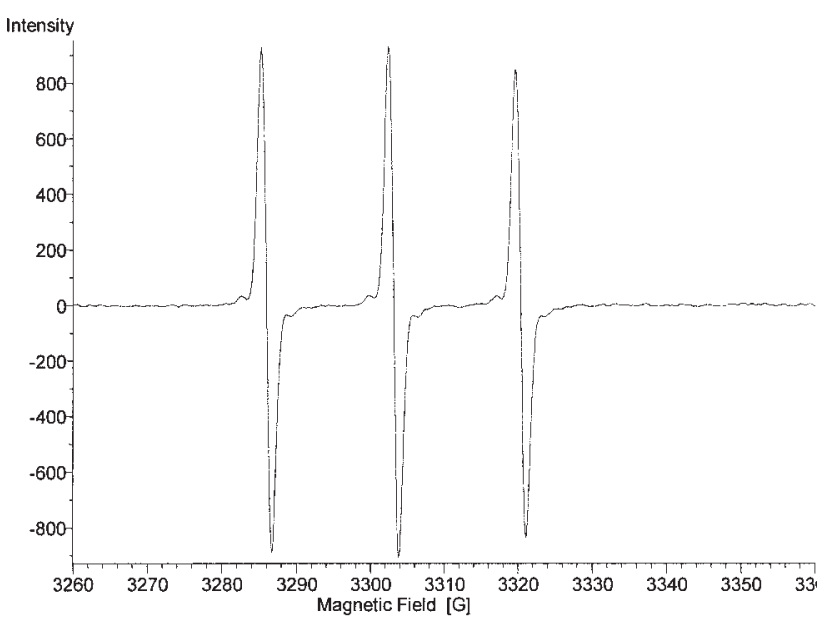

Figure 9 ESR spectrum of liquid after experiment.

vent molecules farther into the center of the lens, making the diffusion of the solvent more and more difficult in the deeper parts of the lens.

The third region was the last $45-50 \mathrm{~min}$, and the lens was saturated with liquid. Hence, the nitroxide radicals could move without restriction. And they began to be transported by the liquid out of the lens. It was deduced that this reversal motion was a process of desorption. To investigate the validity of this idea, some liquid was taken from the tube at the end of the experiment $(t=300 \mathrm{~min})$. We put this liquid into another ESR tube and took the ESR spectrum to test if it had any nitroxide radicals. We know there were no radicals in the liquid at the beginning of the experiment $(t=0)$. But now it had an ESR spectrum like that shown in Figure 9. This proved the reversal motion or desorption process, explained as above.

The value of $2.50 \times 10^{-8} \mathrm{~cm}^{2} / \mathrm{s}$ was greater than that of the second region, $0.35 \times 10^{-8} \mathrm{~cm}^{2} / \mathrm{s}$. This is because of the increased free motion of nitroxide radicals relative to that of the second region. It was smaller than the first region value of $6.38 \times 10^{-8}$ $\mathrm{cm}^{2} / \mathrm{s}$. This behavior could be explained as follows: in the first region only liquid molecules entered into the solid contact lens, whereas in the third region both liquid and nitroxide radicals entered. As a result, the transported liquid molecules were larger when mixed with nitroxide radicals. Therefore, the rate of diffusion was smaller than that observed in the first region.

\section{References}

1. Thimmegowda, M. C.; Sathyanarayana, P. M.; Shariff, G.; Ashalatha, M. B.; Ramani, R.; Ranganatharah, C. Phys Stat Sol 2002, 2, 257.

2. Brown, I. M.; Sandreczki, T. C. Macromolecules 1983, 16, 1890. 
3. Demir, Y. Ph.D. Thesis, Hacettepe University, Ankara, Turkey, 1987.

4. Veksli, Z.; Miller, W. G. Macromolecules 1977, 10, 1245.

5. Veksli, Z.; Miller, W. G. J Polym Sci 1976, 54, 299.

6. Snipes, W.; Keith, A. Research/Development, Feb. 22-26, 1970.

7. Kaptan, H. Y.; Pekcan, Ö.; Arca, E.; Güven, O. J Appl Polym Sci 1989, 37, 2577.

8. Kaptan, H. Y.; Pekcan, Ö.; Güven, O. J Appl Polym Sci 1992, 44, 1595.

9. Kaptan, H. Y. Ph.D. Thesis, Hacettepe University, Ankara, Turkey, 1987.

10. Katırcioglu, Y.; Kaptan, H. Y.; Güven, O. 7th Tihany Symposium on Radiation Chemistry; Balatonszeplak, Hungary, 1990; p 377.
11. Crank, F. The Mathematic in Diffusion; Oxford University Press: London, 1970.

12. Özgür, E. E. Ms.C. Thesis, Hacettepe University, Ankara, Turkey, 2003.

13. Eken, M.; Turhan, Ş.; Kaptan, H. Y.; Güven, O. Radiat Phys Chem 1995, 46, 809.

14. Berens, A. R.; Hopfenberg, H. B. Polymer 1978, 19, 489.

15. Kaptan, H. Y. J Appl Polym Sci 1999, 71, 1203.

16. Randby, B.; Rabek, J. E. ESR Spectroscopy in Polymer Research; Springer-Verlag: New York, 1977.

17. Berliner, L. J. Spin Labeling: Theory and Applications; Academic Press: New York, 1976. 